\title{
SYNTHESIS OF REAL-TIME CONTROL SYSTEMS FOR MULTILINK INDUSTRIAL ROBOTS BASED ON HYBRID NEURAL NETWORK APProach OF Solution InVERSE Kinematics Problem
}

\author{
Pavel Ganin, Aleksandr Kobrin, Denis Shilin, Valery Moskvin \& Dmitry Shestov
}
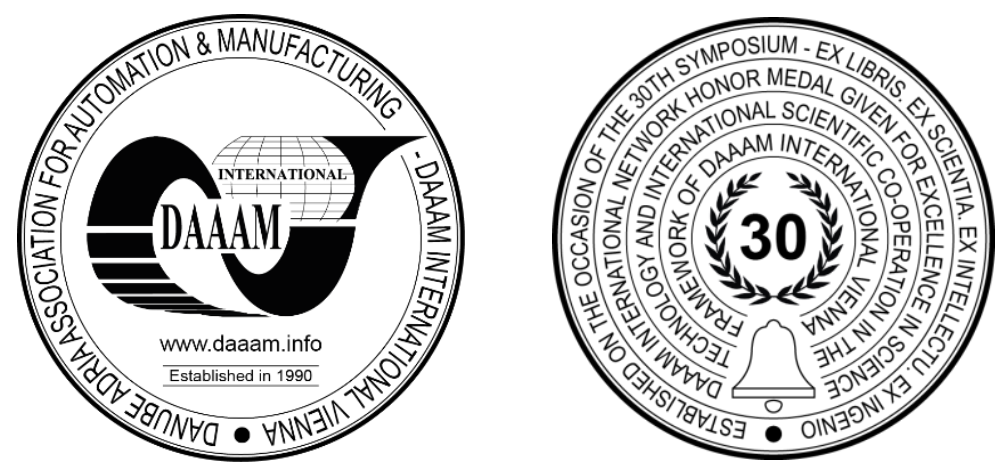

This Publication has to be referred as: Ganin, P[avel]; Kobrin, A[lexandr]; Shilin, D[enis]; Moskvin, V[alery] \& Shestov, D[mitrii] (2019). Synthesis of Real-Time Control Systems for Multilink Industrial Robots Based on Hybrid Neural Network Approach of Solution Inverse Kinematics Problem, Proceedings of the 30th DAAAM International Symposium, pp.0513-0517, B. Katalinic (Ed.), Published by DAAAM International, ISBN 978-3-902734-22-8, ISSN 1726-9679, Vienna, Austria

DOI: $10.2507 / 30$ th.daaam.proceedings.069

\begin{abstract}
The paper considers the issue of constructing real-time control systems based on a new developed hybrid method for solving the inverse kinematics problem. A method based on neuro-fuzzy network (ANFIS) with subsequent iterative refinement of the obtained solution by Newton-Raphson numerical method is proposed. Studies of the applicability of this method in control systems for three-, five- and eight-link robotic manipulators structures are carried out. The comparative analysis of methods for solution of IK problem: classical iterative, method based on neural network and the proposed hybrid is presented. The possibility of adaptation of neural networks for the developed IK method using a corrective buffer is considered. The solution as the system of equations for direct kinematics of multi-link structures is given.
\end{abstract}

Keywords: robot; inverse kinematics; real time control system; ANFIS; iterative refinement.

\section{Introduction}

Constructing a control system involves finding a compromise between conflicting requirements. The complexity of the system synthesis depends on the large dimension of its mathematical model (a high order of differential equations describing it) and often the impossibility of an exhaustive analytical representation. In General, the control system should provide a stable and predictable operation of the robot manipulator under conditions of invariance to external influences. With regard to manipulative robots operating in a dynamically changing environment, the following problems of control system synthesis can be highlighted:

- complexity and multi-connectivity of the system;

- the need for real-time calculations;

- change of control parameters (due to wear) during long-term operation;

- presence of the input saturation due to the structural actuators limitations. 
To solve these problems, a large number of different approaches have been developed. An example is the principle of decomposition of a complex manipulator control system on a number of smaller subsystems of each link [1]. This approach greatly simplifies the synthesis of control systems, but eliminates the consideration of mutual influence when considering the system as a whole. In addition, the construction of control systems based on recurrent decomposition is considered in the concept of industry 4.0 [2]. The principle of decomposition is the basis of the developed real-time control system and algorithms for solving the inverse kinematics of the robot manipulator. The proposed architecture for the control system of an industrial robot based on the Festo stepper motors is presented in more detail in [3].

The article proposes a new method for solving problems related to the determination of the manipulator variables (the angles of links rotation) at a given position of the end-effector - that is, with the solution of the inverse kinematic problem of the manipulator. Methods for solving the IK problem in general are divided into exact and approximate ones. With the use of exact methods, the desired rotation angles can be obtained in the form of an analytical dependence of the parameters of the kinematic scheme of the manipulator. In this case, the process of finding the desired angles is reduced to calculating the values of the previously obtained analytical dependences. Unfortunately, the exact solution can not be obtained for each kinematic scheme of the manipulator. To solve this problem, approximate methods are used - methods of numerical solutions of the coupling equations. Such methods, in principle, make it possible to find solutions for any kinematic schemes, but these methods have a number of disadvantages due to the use of an iterative approach to the solution. Currently, in science and technology there is a tendency to increase the adaptability and intelligence of algorithms, the possibility of self-learning for the control system. The most perspective ways of control systems synthesis for industrial robots are those based on the use of neural networks. Trained neural networks can greatly simplify the process of finding solutions to IK problem, they have the ability to adapt for solving typical problems. Applying neural networks for solving IK problem, in contrast to analytical approaches, where it is required to specify strict analytical dependencies, is possible for various constructions of manipulators. By combining the human-like style of reasoning of fuzzy systems with training and connectionist structure of neural networks, neuro-fuzzy networks were created. The main advantage of fuzzy neural networks is their ability to approximate and provide the possibility of application in universal control systems due to learning algorithms. Adaptability and flexibility of such networks can greatly simplify the construction of control systems. Studies in the field of adaptive technologies and methods of artificial intelligence are based on the works of such specialists as O. Bologa [4], I. Breido [5], O. Castillo [6], F. L. Chernousko [7], E. L. Eremin [8], J. S. R. Jang [9], B. C. Nakra [10], A. Singla [11], A. S. Yushchenko [12] and others.

\section{Kinematics of robot manipulator}

The considered robot-manipulator consists of series-connected links, the first of which is connected to the base, and the last is provided with a working tool (gripper). Each of the links has connections with no more than two others so that no closed circuits are formed. The connection of two links is a joint that has only one degree of freedom (rotation). The links of the manipulator participate in the relative motion, as a result of which a certain position and orientation of the end-effector in space is achieved. Depending on the application of the manipulator and the tasks, a diagram of the mechanical structure is constructed, it determines the parameters of all links and joints. Control system synthesis of robots involves solving direct and inverse kinematic problems and constructing the workspace area. The direct kinematics is to determine the spatial position and orientation of the characteristic point (manipulator tool) by the known values of the generalized coordinates. We consider the robot-manipulator for which the system of equations describes its kinematics has the following form:

$$
\left\{\begin{array}{c}
x=\left[\sum_{m=1}^{n}\left(L_{m} \cdot \sin \left(\sum_{p=1}^{m} Q_{p}\right)\right)\right] \cdot \cos Q_{0}, \\
y=\left[\sum_{m=1}^{n}\left(L_{m} \cdot \sin \left(\sum_{p=1}^{m} Q_{p}\right)\right)\right] \cdot \sin Q_{0} \\
z=L_{0}+\sum_{m=1}^{n}\left(L_{m} \cdot \cos \left(\sum_{p=1}^{m} Q_{p}\right)\right)
\end{array}\right.
$$

One of the most important parameters of the manipulator is its workspace - this is the area at each point of which it is possible to position the end-effector of the manipulator. Based on the solution of the direct problem and using the system of equations (1), it is possible to construct the manipulators workspace. The obtained data is used to construct the algorithm for finding the solution of the IK problem.

\section{New hybrid method for solving IK problem}

The method of solving IK problem for the selected group of industrial manipulators described by equations (1) is based on the application of adaptive neuro-fuzzy inference system (ANFIS). Main principle of operation is the sequential application of the neural network and the numerical implementation of the Newton-Raphson iterative method to refine the obtained approximate value.

Figure 1 shows the scheme of the developed hybrid method for solving IK problem. For a given target position of the end-effector of the manipulator in space $(x, y, z)_{T}$, first of all, the reachability check is performed (entering in the working area). Then the target coordinates in space are transferred to the trained neural networks $\left(N N_{1}-N N_{n}\right.$, corresponding to the number of required angles). 
As a result of the initiation of the neural networks, we obtain the approximate value of links angles $\left(Q_{0}-Q_{n}\right)_{N N}$. By solving the direct kinematics $f\left(Q_{0}-Q_{n}\right)_{N N}$ the control system checks the accuracy of the obtained IK solution, and in case of its insufficiency proceeds to numerical iterations in the locality of the approximate values. When the required accuracy is achieved, the solution $\left(Q_{0}-Q_{n}\right)_{R}$ is transferred to the coordinate controller, which, in turn, monitors the execution of movements. The control signal is sent to the electric stepper motors by the servo controller. During operation of the manipulator, the correction buffer $B u f$ is generated (adjusted data $(x, y, z)_{R}=f\left(Q_{0}-Q_{n}\right)_{R}$ from iterative refinement algorithm). In the future, it is used to adjust the training samples for neural networks and correct them.

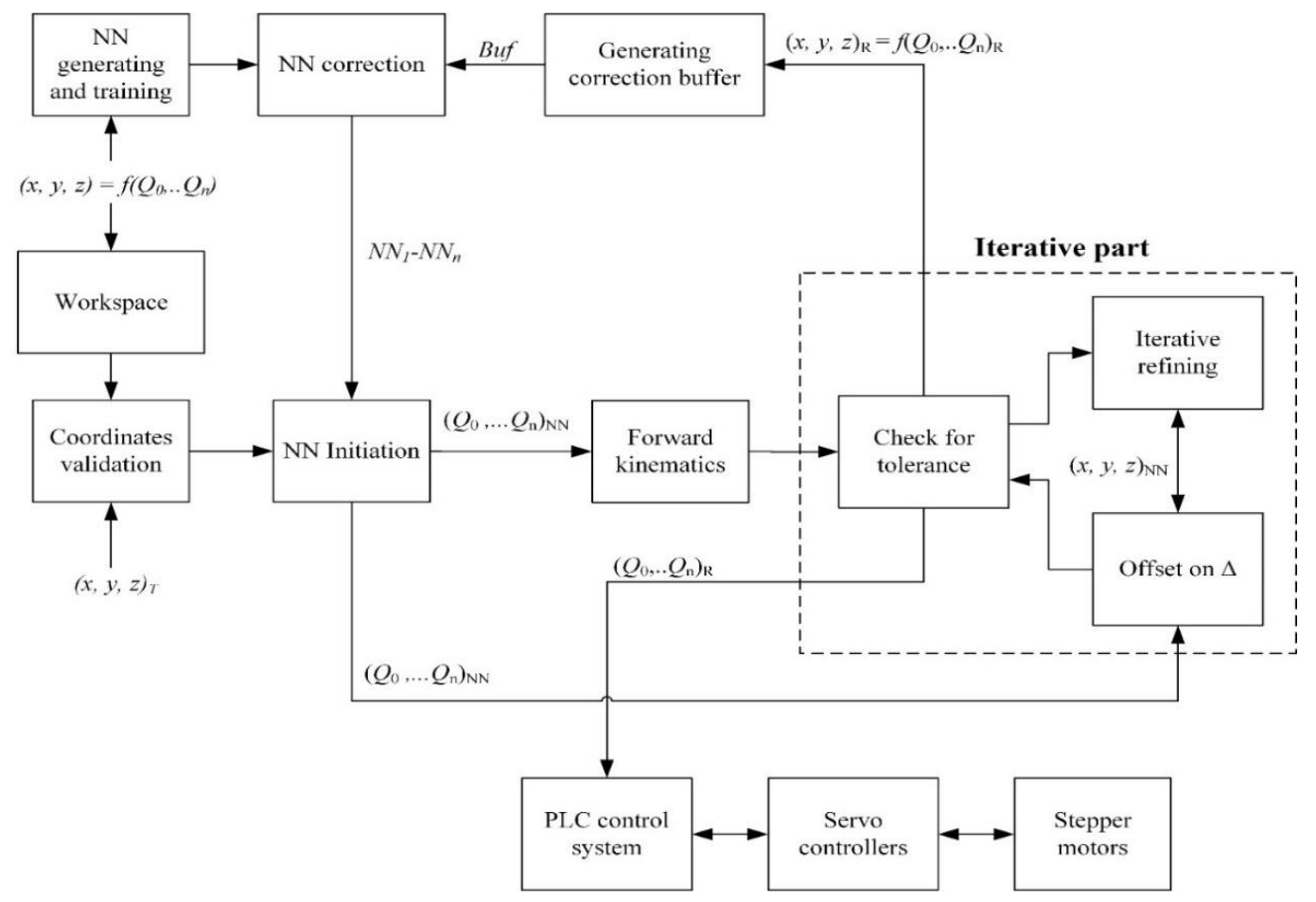

Fig. 1. The scheme of the hybrid method for IK problem

The main advantage of the described method is to increase the performance of the algorithm calculations with controlled accuracy. It should be noted that all numerical iterative methods are very resource-intensive and require a large number of iterations, which in turn negatively affects the algorithm execution time. On the other hand, methods for solving IK problem based on the use of neural networks are performed much faster (2-5 [ms] for point) and do not depend on the construction complexity (systems of equations which describe it). However, the accuracy of calculations of neural network methods is not very high in comparison with iterative ones. Therefore, we propose an algorithm that combines the advantages of both methods: high accuracy and performance. The solution for IK problem is first calculated by a fuzzy neural network, and then refined by the Newton-Raphson iterative method. This significantly reduces the number of iterations of the numerical method (the execution time of the algorithm). The use of neuro-fuzzy network guarantees singular solution of the IK problem and provides high performance. Neural networks also provide high possibilities of adaptation to different robot constructions and tasks. It should be noted that it is necessary to generate and train the networks previously, and this process requires a long time.

\section{Experimental analysis of the developed hybrid IK method}

The article presents the results of experimental studies of the developed hybrid method for solving IK problem for multi-link industrial manipulators. A comparative analysis of iterative, neural network and hybrid methods is performed. A series of experiments were carried out, the input data for which were linear arrays of points corresponding to the desired closed trajectories of the end-effector. All trajectories are strictly inside the manipulators working area and exclude going beyond its boundaries. The selection of trajectories and their construction were performed taking into account the coverage of most of the working area of the manipulator and included consideration of its possible singular positions. Coordinates calculation for the manipulators end-effector were performed on the set of trajectories by various methods (iterative, neural network and hybrid). The maximum, minimum and average (median) deviation of the manipulators tool from a target point is calculated. The time spent by the control system for neural network generation, its training and calculation of coordinates is also calculated. The accuracy of position calculation for the working tool of the manipulator is set to $1[\mathrm{~mm}]$. The experimental data are presented in table 1, it shows that the time for calculating the coordinates for one point does not exceed 3 [ms]. The maximum total deviation does not exceed the specified refinement accuracy. 


\begin{tabular}{|c|c|c|c|c|c|c|c|c|c|c|}
\hline \multirow{3}{*}{\multicolumn{2}{|c|}{ Tolerance 1 [mm] }} & \multirow{2}{*}{\multicolumn{3}{|c|}{$\begin{array}{c}\text { Iterative method } \\
\text { links: }\end{array}$}} & \multirow{2}{*}{\multicolumn{3}{|c|}{$\begin{array}{c}\text { Neural Network } \\
\text { links: }\end{array}$}} & \multirow{2}{*}{\multicolumn{3}{|c|}{$\begin{array}{c}\text { Hybrid method } \\
\text { links: }\end{array}$}} \\
\hline & & & & & & & & & & \\
\hline & & 3 & 5 & 8 & 3 & 5 & 8 & 3 & 5 & 8 \\
\hline \multicolumn{2}{|c|}{ Time for trajectory calculation, [ms]: } & 10311 & 64210 & 158699 & 3940 & 6213 & 9947 & 4381 & 7058 & 11083 \\
\hline \multicolumn{2}{|c|}{ Time for calculation of one point, [ms]: } & 2.578 & 16.053 & 39.675 & 0.985 & 1.553 & 2.487 & 1.095 & 1.765 & 2.771 \\
\hline \multirow{3}{*}{$\begin{array}{c}\text { Number of } \\
\text { iterations per } \\
\text { point, [pcs] }\end{array}$} & min: & 13 & 16 & 28 & & & & 11 & 12 & 12 \\
\hline & med: & 139 & 2634 & 11876 & & & & 13 & 15 & 16 \\
\hline & max: & 11429 & 42296 & 71871 & & & & 60 & 72 & 157 \\
\hline \multirow{3}{*}{$\begin{array}{l}\text { Distance from the } \\
\text { end-effector to the } \\
\text { target, }[\mathrm{mm}]\end{array}$} & min: & 0.026 & 0.158 & 0.344 & 11.475 & 21.608 & 46.920 & 0.096 & 0.608 & 0.827 \\
\hline & med: & 0.868 & 0.947 & 0.963 & 16.604 & 25.786 & 65.720 & 0.814 & 0.917 & 0.958 \\
\hline & max: & 1.000 & 1.000 & 1.000 & 21.713 & 28.112 & 82.257 & 1.000 & 1.000 & 1.000 \\
\hline
\end{tabular}

Table 1. Comparative analysis of IK methods

The following conclusions can be drawn from the data obtained (table 1):

- when using the neural network method, the best performance of the system is achieved, but the average deviation (distance to the target) is much higher (17, 26 and 66 times depending on the number of links) than the established tolerance;

- the time for coordinates calculation with use of the iterative method is significantly increased (by $623 \%$ and $1539 \%$ for five and eight structures in relation to three-link) with an increase in the number of links;

- duration of calculation by neural network and hybrid methods has no significant dependence on the number of links;

- the hybrid method achieves a significant increase in performance (reduction of the calculation time by $2.5,10$ and 16 times in comparison with the neural network method for the corresponding number of links) with controlled accuracy.

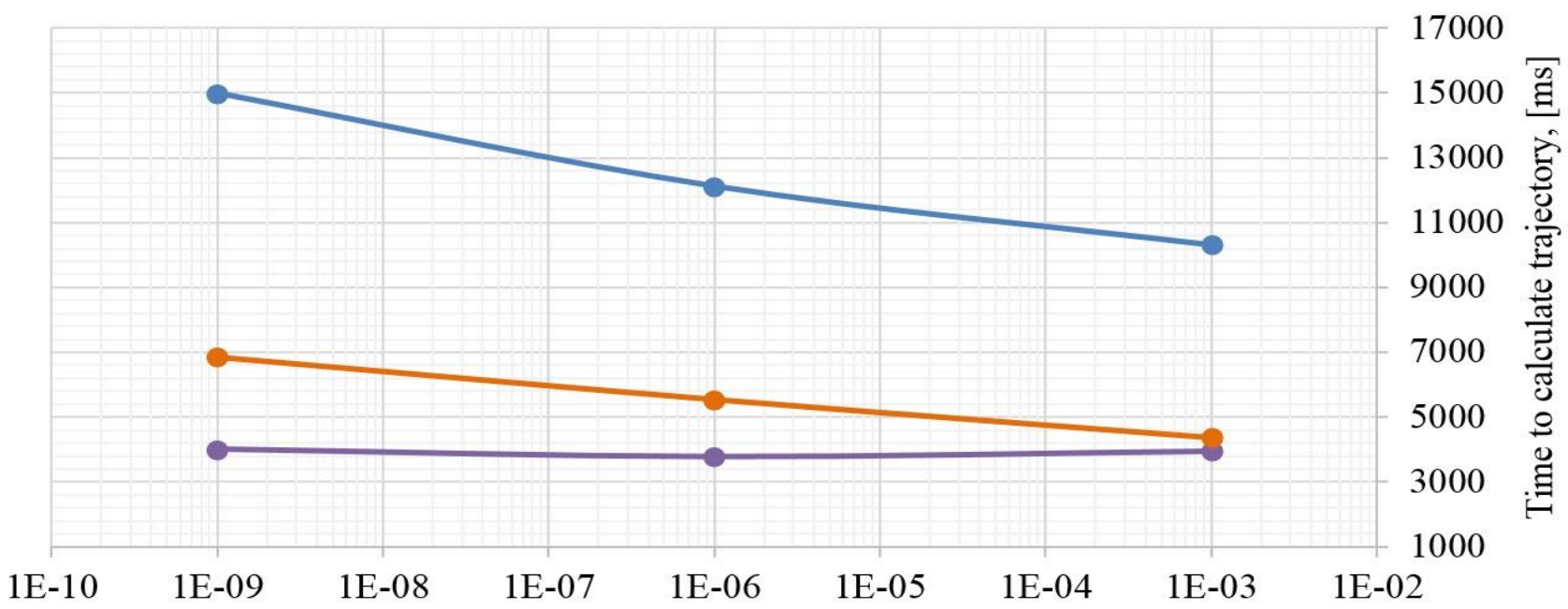

Accuracy of calculations, $[\mathrm{mm}]$

$\longrightarrow$ Iterative method $\longrightarrow$ Neural network method $\rightarrow$ New hybrid method

Fig. 2. The scheme of the hybrid method for IK problem

Fig. 2 shows the graphical dependence of the duration of the solution search for a given trajectory on the required established accuracy for the three-link manipulator. Analyzing the presented data, it can be concluded that the duration of the solution search is proportional to the required accuracy of calculations when using iterative method, and when using neural networks or for the proposed hybrid method the influence of the target tolerance is insignificant.

\section{Adaptation of hybrid IK method}

The developed method for finding IK solution provides training of neural networks in the course of manipulators operation on the acquired refined data. Thus, the algorithms are adapted to perform the same type of tasks. Table 2 is presented with the results of experiments in the calculation of the IK problem for three-, five - and eight-links industrial manipulators with the established tolerance of calculations of $1[\mathrm{~mm}]$ for the position of the working tool. The calculations are presented for the developed hybrid method and its adapted version, adjusted on the basis of the data obtained after the refinement calculations. 


\begin{tabular}{|c|c|c|c|c|c|c|c|}
\hline \multirow{3}{*}{\multicolumn{2}{|c|}{ Tolerance 1 [mm] }} & \multirow{2}{*}{\multicolumn{3}{|c|}{$\begin{array}{c}\text { Non-Adopted method } \\
\text { links: }\end{array}$}} & \multirow{2}{*}{\multicolumn{3}{|c|}{$\begin{array}{c}\text { Adopted method } \\
\text { links: }\end{array}$}} \\
\hline & & & & & & & \\
\hline & & 3 & 5 & 8 & 3 & 5 & 8 \\
\hline \multicolumn{2}{|c|}{ Time for trajectory calculation, [ms]: } & 4381 & 7058 & 11083 & 2743 & 3415 & 5002 \\
\hline \multicolumn{2}{|c|}{ Time for calculation of one value, [ms]: } & 1.095 & 1.765 & 2.771 & 0.686 & 0.854 & 1.251 \\
\hline \multirow{3}{*}{$\begin{array}{c}\text { Number of iterations per } \\
\text { point, [pcs] }\end{array}$} & min: & 11 & 12 & 12 & 3 & 6 & 11 \\
\hline & med: & 13 & 15 & 16 & 8 & 10 & 12 \\
\hline & max: & 60 & 72 & 157 & 36 & 46 & 55 \\
\hline
\end{tabular}

Table 2. Hybrid method adaptation

From the presented results (table 2) it can be concluded that the application of adaptation for the hybrid method of IK calculation significantly (reduction from 25 to $40 \%$ depending on the number of links) reduces the number of required cycles of iterative refinement and, as a consequence, the time spent. Moreover, with increasing complexity of the construction (increasing the number of links), the effect of adaptation is most expressed. A reduction in the calculation time of one point by 37,52 and $55 \%$ for three-, five - and eight-links robot-manipulators construction was noted.

\section{Conclusion}

The constructing a control system involves finding a compromise between conflicting requirements: ensuring high accuracy of calculations at the lowest possible time. It is also necessary to provide the possibility of calculation algorithms adaptation in the conditions of changing parameters. To solve these problems, a new hybrid method for solving IK problem for multilink kinematically redundant manipulator based on neuro-fuzzy networks and iterative refinement of the numerical solution is developed. The presented results of the study demonstrate an increase in the performance of IK problem calculation algorithms in comparison with the classical iterative and neural network approaches. The increase of performance for applying adaptation of calculation algorithms is noted. The presented experiments are considered strictly for a certain group of manipulators mechanical structures.

The presented method of solving IK problem can be effectively applied in combining with trajectory planning algorithms for kinematically redundant manipulators. It is planned to conduct research for applying in couple with planning algorithms. In the future, it is also planned to analyze the application of the developed method for other types of manipulator structures, taking into account the end-effector orientation.

\section{References}

[1] Pyatnitsky, E. S. (1989). Synthesis of hierarchical control systems of mechanical and Electromechanical objects on the principle of decomposition. Automation and telemechanics, Vol. 1, pp. 87-98.

[2] Yerofeyev, S; Ipatov, O; Markov, S; Potekhin, V; Sulerova, A \& Shkodyrev, V. (2016). Adaptive Intelligent Manufacturing Control Systems, Proceedings of the 26th DAAAM International Symposium, pp.1016-1024, B. Katalinic (Ed.), Published by DAAAM International, ISBN 978-3-902734-07-5, ISSN 1726-9679, Vienna, Austria.

[3] Ganin, P; Kobrin, A; Belousov, M \& Shilin, D. (2015). Simulation Manipulator Based on Step Motors Festo, Chapter 11 in DAAAM International Scientific Book 2015, pp.127-134, B. Katalinic (Ed.), Published by DAAAM International, ISBN 978-3-902734-05-1, ISSN 1726-9687, Vienna, Austria.

[4] Crenganis, M.; Breaz, R.; Racz, G. \& Bologa, O. (2014). Inverse kinematics for a 7 DOF robotic arm using the redundancy circle and ANFIS models. Applied Mechanics and Materials, No. 657, pp. 823-828.

[5] Breido, I \& Shpakova, L. (2017). Analysis of the Application of Fuzzy Logic Methods for the Robot-Manipulator Control system, Chapter 10 in DAAAM International Scientific Book 2017, pp.121-132, B. Katalinic (Ed.), Published by DAAAM International, ISBN 978-3-902734-12-9, ISSN 1726-9687, Vienna, Austria.

[6] Melin, P. \& Castillo, O. (2005). Intelligent control of a stepping motor drive using an adaptive neuro-fuzzy inference system. Information Sciences, No. 170 (2-4), pp. 133-151.

[7] Chernousko, F. L.; Ananyevsky, I. M.; Reshmin, S. A. (2006). Methods of control of nonlinear mechanical systems. M .: Fizmatlit, 328 p.

[8] Eremin, E. L. \& Shelenok, E. A. (2018). Synthesis of the Decentralized Control System for Robot-Manipulator with Input Saturations. IT in Industry, Vol. 6, pp. 27-32. ISSN: 2204-0595.

[9] Jang, J. S. R. (1993). ANFIS: adaptive-network-based fuzzy inference system. IEEE Transactions on Systems, MAN, and Cybernetics, Vol. 23, No.3, pp. 665-685.

[10] Manjaree, S.; Nakra, B.C. \& Agarwal, V. (2015). Comparative analysis for kinematics of 5-DOF industrial robotic manipulator. Acta Mechanica et Automatica, Vol. 9 (4), pp. 229-240.

[11] Narayan, J. \& Singla, A. (2017). ANFIS based kinematic analysis of a 4-DOFs SCARA robot. 4th IEEE International Conference on Signal Processing, Computing and Control, ISPCC 2017, pp. 205-211.

[12] Yushchenko, A. S. \& Mikhailov, B. B. (2004). Interactive control of mobile robots using fuzzy logic. Mechatronics, automation, control, No.6, pp. 24-31. 